

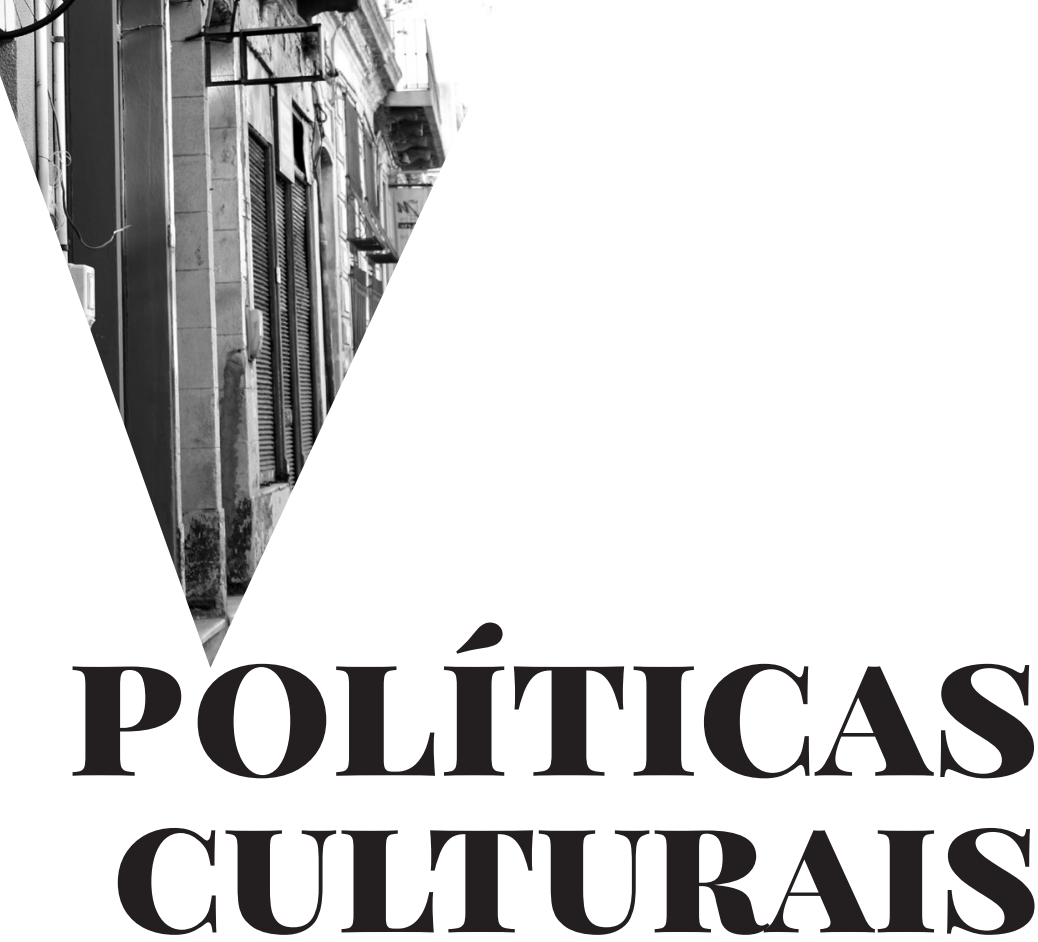

\section{em Revista}

\begin{tabular}{|l|l|l|l|l|l|l|}
\hline Pol. cult. rev. & Salvador & v. 12 & n. 2 & p. 1-253 & jul./dez. & 2019 \\
\hline
\end{tabular} 


\title{
UNIVERSIDADE FEDERAL DA BAHIA
}

REITOR

João Carlos Salles Pires da Silva

VI CE-REITOR

Paulo César Miguez de Oliveira

\author{
Instituto de Humanidades, Artes e Giências \\ Professor Milton Santos \\ DI IREÇÃO \\ Messias Guimarães Bandeira
}
Programa Multidisciplinar de Pós-Graduação em Cultura e Sociedade
CoOrdenação
José Roberto Severino

Centro de Estudos Multidisgiplinares em Cultura

\author{
COORDENAÇÃo \\ Adriano Sampaio \\ Vice-CoORdENAD ORA \\ Lynn Alves
}

EDITORES-CHEFES

Alexandre Barbalho, Universidade Estadual do Ceará

Leonardo Costa, Universidade Federal da Bahia

Renata Rocha, Universidade Federal da Bahia

Editores do dosiê Políticas Culturais e Democracia

José Márcio Barros, Universidade do Estado de Minas Gerais

Lia Calabre, Fundação Casa de Rui Barbosa

Conselho Editorial

Alain Herscovici, Universidade Federal do Espírito Santo

Ana Carolina Escosteguy, Pontíficia Universidade Católica do Rio Grande do Sul

Ana Rosas Mantecón, Universidade Autónoma Metropolitana do México

Armand Mattelart, Universidade Paris VIII 
Carlos Lopes, United Nations Institute for Training and Research

Carlos Yáñez Canal, Universidad Nacional de Colombia

César Bolaño, Universidade Federal de Sergipe

Daniel Mato, Universidad Central de Venezuela

Durval Albuquerque, Universidade Federal do Rio Grande de Norte

Emir Sader, Universidade do Estado do Rio de Janeiro

Fabio de Castro, Universidade Federal do Pará

George Yúdice, University of Miami

Guilhermo Sunkel, Victoria University, Austrália

Guillermo Mariaca Iturri, Universidad Mayor de San Andrés

Gustavo Lins Ribeiro, Universidade de Brasília

José Machado Pais, Universidade de Lisboa

Lúcia Lippi, Fundação Getúlio Vargas

Manuel Garretón, Universidad de Chile

Marcelo Ridenti, Universidade Estadual de Campinas

Maria de Lourdes Lima Santos, Universidade de Lisboa

Muniz Sodré, Universidade Federal do Rio de Janeiro

Octavio Getino, Instituto Universitário Nacional de Artes da Argentina

Renato Ortiz, Universidade Estadual de Campinas

Rubens Bayardo, Universidad San Martin - Universidad de Buenos Aires

Xan Bouzadas, in memorian

Conselho de Redaçä́o

Alexandre Barbalho, Universidade Estadual do Ceará

Antonio Albino Canelas Rubim, Universidade Federal da Bahia

Anita Simis, Universidade Estadual Paulista

Cláudia Leitão, Universidade Estadual do Ceará

Cristina Lins, Instituto Brasileiro de Geografia e Estatística

Humberto Cunha, Universidade de Fortaleza

Isaura Botelho, Centro Brasileiro de Análise e Planejamento

José Márcio Barros, Pontifícia Universidade Católica de Minas Gerais - Universidade do Estado

de Minas Gerais

Leonardo Costa, Universidade Federal da Bahia

Lia Calabre, Fundação Casa de Rui Barbosa

Maria Helena Cunha, DUO Informação e Cultura

Paulo Miguez, Universidade Federal da Bahia

NormalizaÇÃo, ReVis Ão e DiagramaÇÃo:

Equipe EDUFBA 


\section{Sumário}

Políticas Culturais e Demogracia 8

José Marciơ Barross, Lia Cafabre

Construindo a Democracia Gultural:

Gidadania, Federação e Participação 13

Marcelo Viana Estevão de Moraes

Política Gultural no Brasil:

RETROGESSOS, RESISTENCIA E REEXISTENCIA 34

Tárcio Mota, Osmar Moreira

Participaçäo sogial nas políticas culturais: O Conselho

Nacional de Políticas Gulturais e o contexto de crise DEMOGR ÁTICA 5 O

Mariana de Araujo Aguiar, Júlia Erminia Riscado

A frágil democracia BRASILEIRA E OAL ESTAR SOFRido

NO ÂMBITO CULTURAL: Os DESAFIOS DO MINC

E DAS POLÍticas CULTURAis 72

Olívia dos Santos Nascimento, Tamiles Alves

A MANifESTA COMO UM EXPERIMENTO

DE PARTICIPAÇÃO SOGIAL 87

Viviane Pinto, Cayo Honorato

Artigos 109

LA IMPLEMENTACIÓN DE LAS POLITICAS CINEMATOGRÁFICAS EN COLOMBIA Y LOS CAMBIOS EN LOS ARREGLOS

ORGANIZACIONALES 11 O

Liceth Viviana Rivera Mancilla, Mariana Baldi

Sobre la MULTiplicidad de lo múltiple:

LA GESTIÓN CULTURAL EN LATINOAMÉRICA 130

Carlos Yáñez Canal 
O Palan que: Artistas na campanha das diretas Já 145

Fabio Maleronka Ferron

Produçä́o artística, democracia e Estado:

REFLEXÕES SOBRE DIFERENTES PERSPECTIVAS GOVERNAMENTAIS PARA AS ARTES 163

Heloisa Marina

Participação Sogial e institucionalizaçä́:

de políticas setoriais de cultura no Rio de Janeiro 190

Juliano Borges, Simone Amorim

Contradiçöes, limites e possibilidades: a experiência da Lei Municipal de Incentivo à Cultura de Belo Horizonte NO FOMENTO À PRODUÇÃO AUDIOVISUAL DA PERIFERIA 211

Marcelo Braga de Freitas

DiREITOS GULTURAIS：DE ONDE FALAMOS?

PARA AONDE PRETENDEMOS IR? 236

Naiene Sanchez Silva 


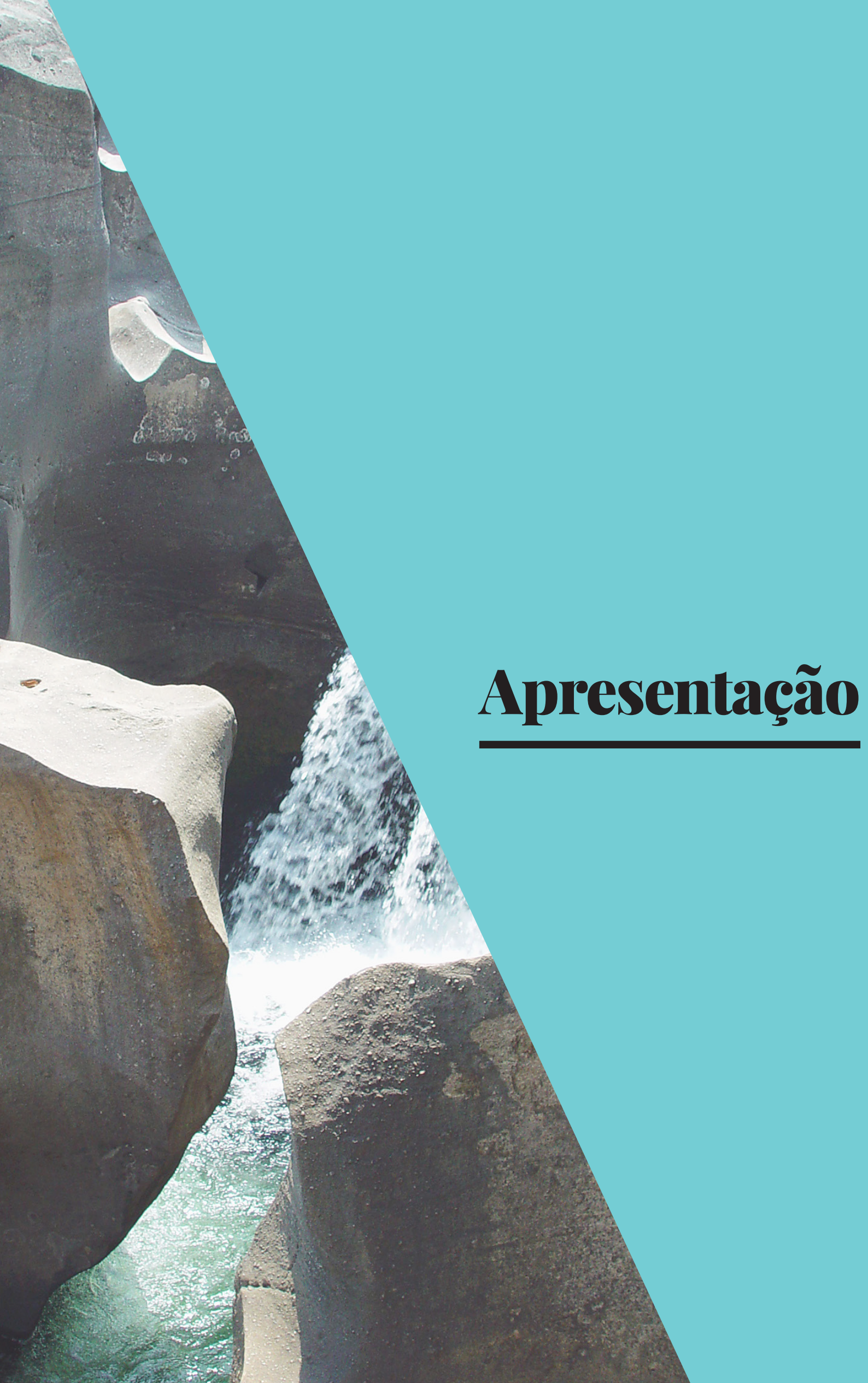




\section{Políticas culturais e democracia}

Jơsé Marciơ Barrơs ${ }^{1}$ e Lia Calabre ${ }^{2}$

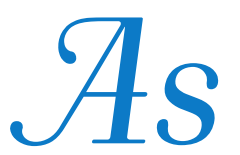

relações entre as políticas culturais e a democracia são amplas e complexas, exigindo, do campo de estudos e ação, constantes atualizações conceituais. Democracia, como afirma Marilena Chauí, não se reduz a um modelo de governo, mas se refere à forma de organização e funcionamento de uma sociedade em suas diversas dimensões e interações. Excede, portanto, a um sistema de representação política ancorado em processos eleitorais e rodízio de governantes, consubstanciando-se como um regime de organização política, social e econômica

1 Doutor em Comunicação e Cultura pela Universidade Federal do Rio de Janeiro, professor do Programa de Pós-Graduação em Artes da Universidade do Estado de Minas Gerais e do Programa de Pós-Graduação em Cultura e Sociedade da Universidade Federal da Bahia. Coordenador do Observatório da Diversidade Cultural (ODC), josemarciobarros@gmail.com

2 Doutora em História, coordenadora da Cátedra Unesco de Políticas Culturais e Gestão, da Fundação Casa de Rui Barbosa (FCRB), professora do Programa de Pós-Graduação em Memória e Acervos-FCRB e do Programa de Pós-Graduação Cultura e Territorialidades, da Universidade Federal Fluminense, liacalabre@gmail.com 
baseado na liberdade, na isonomia, na participação, no exercício pleno dos direitos e na distinção entre poder e governo. Já as políticas culturais podem ser definidas como um conjunto de princípios, objetivos e ações protagonizado pelo poder público e pela sociedade civil, em seus diversos setores e segmentos, de forma a garantir os direitos culturais.

Em contextos democráticos, tais políticas são construídas e operacionalizadas tendo como base a participação social, a utilização de mecanismos de escuta e deliberação pública, de forma a garantir o exercício pleno da cidadania cultural.

No Brasil e em vários outros países, o protagonismo político, econômico e social da cultura coloca em destaque a relação entre as políticas culturais e a democracia em uma dupla dimensão. Por um lado, a forma como as políticas culturais contribuem para a consolidação da democracia em seu amplo espectro e, por outro lado, a maneira como esta pauta a própria configuração e as práticas daquelas.

A partir do início do século XXI, o país vivenciou um processo de inauguração, aprofundamento e aperfeiçoamento da experiência democrática na elaboração e implementação das políticas públicas. Na esfera federal, a área da cultura ganhou assumidamente status de política pública. Mais do que discutir mecanismos operacionais da experiência democrática, este dossiê busca apresentar e iluminar algumas das questões centrais que podem ou 
não permitir maior efetividade do processo que buscou produzir uma sociedade com políticas públicas mais democráticas, justas e inclusivas.

Neste dossiê, reunimos cinco artigos que desenvolvem análises críticas sobre a realidade vivida no Brasil e que estão ancorados em diferentes perspectivas conceituais, ora evidenciando aspectos que apontam para a importância da cultura no fortalecimento da democracia, ora analisando os efeitos da suspensão dos avanços democráticos no país e a grave descontinuidade que geram nas políticas culturais.

O primeiro artigo, intitulado "Construindo a democracia cultural: cidadania, federação e participação”, de Marcelo Viana Estevão de Moraes, trata da questão da mudança de paradigma da política cultural brasileira a partir do início do século XXI. O autor aprofunda as discussões sobre os fatores essenciais para o sucesso da implementação desse novo modelo e os riscos que as novas orientações do governo federal impõem à ação política consagrada na Constituição Federal de 1988.

O artigo seguinte é "Política cultural no Brasil: retrocessos, resistência e reexistência”, de Tárcio Mota e Osmar Moreira. A partir da reconstituição panorâmica da trajetória das políticas culturais das últimas décadas, os autores apresentam uma reflexão tanto sobre os retrocessos ocorridos no país e seus reflexos nas comunidades quanto sobre as formas de resistência que estas vêm construindo 
para enfrentar os processos de descontinuidade de tais políticas.

O terceiro artigo, de Mariana de Araujo Aguiar e Júlia Erminia Riscado, é "Participação social nas políticas culturais: o Conselho Nacional de Políticas Culturais e o contexto de crise democrática". Nele, as autoras buscam refletir sobre os conceitos de governança e de participação, tendo como objeto de análise histórica os conselhos gestores, através de um recorte que objetiva entender as crises e pensar sobre possíveis perspectivas que se apresentam em um contexto de ameaça aos mecanismos de participação social.

O quarto artigo do dossiê trata da problemática da desinstitucionalização do Ministério da Cultura como um atentado aos direitos culturais. Em "A frágil democracia brasileira e o mal-estar sofrido no âmbito cultural: os desafios do Minc e das políticas culturais”, Olívia dos Santos Nascimento e Tamiles Alves trabalham com a problemática da homogeneização das identidades e dos desrespeitos aos princípios constitucionais que garantem o Estado democrático de direito.

"A Manifesta como um experimento de participação social", de Viviane Pinto e Cayo Honorato, artigo que fecha o dossiê, nos apresenta a experiência da mostra cultural da $3{ }^{\text {a }}$ Conferência Nacional de Juventude. O principal objetivo do artigo é, tendo em vista as alterações radicais de retrocesso que o ambiente político e democrático do 
país vivenciou, revisitar a experiência da Manifesta, refletindo sobre as ideias norteadoras, metodologias, desafios e etapas de desenvolvimento da mostra, assim como sobre os processos de aprendizagem gerados no âmbito da práticas democráticas.

Esperamos que a leitura dos trabalhos contribua não apenas para a elucidação das relações intrínsecas entre democracia e políticas culturais no Brasil, mas essencialmente para reforçar o compromisso de gestores, pesquisadores, artistas e da população em geral com o campo democrático em sua relação com a cultura. 